\title{
Existence of solutions for discrete boundary value problems with second order dependence on parameters
}

\author{
ABoudramane Guiro and IdRISSA Ibrango \\ Laboratoire de Mathmatiques et Informatique (LAMI) \\ Unit de Formation et de Recherche en Sciences et Techniques \\ Universit Nazi Boni \\ 01 BP 1091 Bobo-Dioulasso 01 \\ Bobo Dioulasso, Burkina Faso \\ abouguiro@yahoo.fr, ibrango2006@yahoo.fr
}

\begin{abstract}
We prove the existence of non trivial solution for discrete nonlinear problems of Kirchhoff type. The proof of the main result is based on a mountain pass lemma.

\section{RESUMEN}

Demostramos la existencia de soluciones no triviales para problemas discretos no lineales de tipo Kirchhoff. La demostración del resultado principal está basado en un lema del paso de la montaña.
\end{abstract}

Keywords and Phrases: Kleinian Groups, NEC groups

2010 AMS Mathematics Subject Classification: 47A75; 35B38; 35P30; 34L05; 34L30. 


\section{Introduction}

The aim of this paper is to extend the following discrete boundary value problem studied by Joanna et al in [10] which is subject to certain parameter $w \in W$ :

$$
\left\{\begin{array}{l}
-\Delta\left(|\Delta u(k-1)|^{p(k-1)-2} \Delta u(k-1)\right)+f(k, u(k), w)=0, \quad k \in[1, N] \\
u(0)=u(N+1)=0,
\end{array}\right.
$$

where $N$ is a positive integer with $N \geq 2, \Delta u(k)=u(k+1)-u(k)$ is the forward difference operator, $W$ is some topological space and $[1, N]=\{1,2, \ldots, N\}$. Using the mountain pass lemma the authors in [10] proved an existence of non trivial solutions and gives conditions under which the problem (1.1) has a unique solution. In this paper we deal with the following discrete boundary value problem

$$
\left\{\begin{array}{l}
-\Delta(\mathrm{a}(\mathrm{k}-1, \Delta \mathrm{u}(\mathrm{k}-1)))=\delta(\mathrm{k}) \mathrm{f}(\mathrm{k}, \mathrm{u}(\mathrm{k}), w), \quad \mathrm{k} \in[1, \mathrm{~N}] \\
\Delta \mathrm{u}(0)=\Delta \mathrm{u}(\mathrm{N})=0,
\end{array}\right.
$$

where $a, f, \delta$ are functions to be defined later. Remark that in this paper, we consider another boundary conditions (Neumann boundary condition). Also, the function $a(k-1, \Delta u(k-1))$ which appear in the left-hand side of problem (1.2) is more general than the one which appear in [10. Note that difference equations can be seen as a discrete counterpart of partial differential equations and are usually studied in connection with numerical analysis. In this way, the main operator in problem (1.2)

$$
\Delta(\mathrm{a}(\mathrm{k}-1, \Delta \mathrm{u}(\mathrm{k}-1)))
$$

can be seen as a discrete counterpart of the anisotropic operator

$$
\sum_{i=1}^{N} \frac{\partial}{\partial x_{i}} a\left(x, \frac{\partial}{\partial x_{i}} u\right)
$$

The theory of difference equations occupy a central position in applicable analysis (see [1]-[3] or [6]-[9], [12, 13]) motivated by applications in various biological, physical and chemical models, such has phase-transition, physical processes in which the variable transits from an unstable equilibrium to a stable one... For example, using the three critical points theorem (see [7, Theorem 2.6 ), Candito and D'Agui studied in [4] the existence of three solutions for the problem

$$
\left\{\begin{array}{l}
-\Delta\left(\Phi_{p}\left(\Delta u_{k-1}\right)\right)+q_{k} \Phi_{p}(u(k))=\lambda f\left(k, u_{k}\right), \quad k \in[1, N] \\
\Delta u_{0}=\Delta u_{N}=0
\end{array}\right.
$$

where $\lambda$ is a real positive parameter, $q_{k}>0$ for all $k \in[1, N], \quad \Phi_{k}(s)=|s|^{p-2} s$ with $1<p<+\infty$ and $f:[1, N] \times \mathbb{R} \longrightarrow \mathbb{R}$ is a continuous function. The authors obtain a suitable interval of 
parameters for which problem (1.3) admits constant-sign solutions which are local minimizers of the corresponding Euler-Lagrange functional.

Motivated by the paper [6] and the ideas in [10, we used the mountain pass lemma to prove an existence of a non trivial solution under some hypothesis. With additional conditions we prove the uniqueness of the non trivial solution.

In order to bring evidence our main result, in the Section 2 we deal with some preliminary materials. In Section 3, using the mountain pass lemma, we prove the existence of an non trivial solution and adding some conditions, we study the uniqueness of solution.

\section{Preliminaries}

In the $\mathrm{N}$-dimensional Hilbert space

$$
X=\{x:[0, N+1] \longrightarrow \mathbb{R} ; \text { such that } \Delta x(0)=0=\Delta x(N)\}
$$

with the inner product

$$
(x, y)=\sum_{k=1}^{N+1} \Delta x(k-1) \Delta y(k-1), \quad \forall x, y \in X,
$$

we consider the norm

$$
\|x\|=\left(\sum_{k=1}^{N+1}|\Delta x(k-1)|^{2}\right)^{\frac{1}{2}}
$$

Let the function

$$
p:[0, N] \longrightarrow[2,+\infty)
$$

and denoted by

$$
p^{-}=\min _{k \in[0, T]} p(k) \quad \text { and } \quad p^{+}=\max _{k \in[0, T]} p(k) .
$$

For the data $a, f$ and $\delta$, we assume the following:

$\left(H_{0}\right) . \begin{cases}a(k, .): \mathbb{R} \rightarrow \mathbb{R}, \quad k \in[0, N] \text { and there exists } A(., .):[0, N] \times \mathbb{R} \rightarrow \mathbb{R} \\ \text { which satisfies } a(k, \xi)=\frac{\partial}{\partial \xi} A(k, \xi) \text { and } A(k, 0)=0, \text { for all } k \in[0, N] .\end{cases}$

$\left(H_{1}\right)$. For any $k \in[0, N], \xi \in \mathbb{R}$ there exist a constant $c_{0}>0$ such that

$$
|a(k, \xi)| \leq c_{0}\left(1+|\xi|^{p(k)-1}\right) .
$$

$\left(\mathrm{H}_{2}\right)$. For any $k \in[0, \mathrm{~N}], \xi \in \mathbb{R}$, we have

$$
A(k, \xi) \geq \frac{1}{p(k)}|\xi|^{p(k)} .
$$


$\left(\mathrm{H}_{3}\right)$. For each $k \in[0, \mathrm{~N}]$, the function $f(k, .,):. \mathbb{R} \times W \longrightarrow \mathbb{R}$ is jointly continuous and there exists a constant $c_{1}>0$ and a function $r:[0, N] \longrightarrow(1,+\infty)$ such that

$$
|f(k, \xi, w)| \leq c_{1}\left(1+|\xi|^{r(k)-1}\right)
$$

where the space $W$ is some topological space. We denoting

$$
F(k, \xi, w)=\int_{0}^{\xi} f(k, s, w) d s \text { for }(k, \xi, w) \in[0, N] \times \mathbb{R} \times W
$$

and we deduce that there exist a constant $c_{2}>0$ such that

$$
|\mathrm{F}(\mathrm{k}, \xi, w)| \leq c_{2}\left(1+|\xi|^{\mathrm{r}(\mathrm{k})}\right)
$$

$\left(H_{4}\right) . \quad \lim _{\xi \rightarrow 0} \frac{f(k, \xi, w)}{|\xi|^{p^{+}-1}}=0$ uniformly for all $k \in[1, N], \quad w \in W$.

$\left(\mathrm{H}_{5}\right) . \delta:[0, \mathrm{~N}] \longrightarrow(0,+\infty)$ such that for all $k \in[0, \mathrm{~N}]$,

$$
0<\underline{\delta}=\inf _{k \in[0, N]}(\delta(k)) \leq \delta(k) \leq \bar{\delta}=\sup _{k \in[0, N]}(\delta(k))<+\infty .
$$

$\left(H_{6}\right)$. There exists constants $c_{3}, c_{4}>0$ and $\mu>p^{+}$such that

$$
\mathrm{F}(k, \xi, w) \geq c_{3}|\xi|^{\mu}-c_{4}, \quad \text { for all } \quad k \in[0, N], \quad \xi \in \mathbb{R}, \quad w \in W \text {. }
$$

\section{Example 2.1.}

We give the following functions where a non trivial solution can be estimate as in [10]:

- $A(k, \xi)=\frac{1}{p(k)}|\xi|^{p(k)}$, where $a(k, \xi)=|\xi|^{p(k)-2} \xi, \forall k \in[0, N]$ and $\xi \in \mathbb{R}$,

- $A(k, \xi)=\frac{1}{p(k)}\left(\left(1+|\xi|^{2}\right)^{p(k) / 2}-1\right)$, where $a(k, \xi)=\left(1+|\xi|^{2}\right)^{(p(k)-2) / 2} \xi, \quad \forall k \in[0, N]$, $\xi \in \mathbb{R}$,

- $f(k, \xi, w)=1+|\xi|^{p(k)-1}, \quad \forall k \in[0, N]$ and $\xi \in \mathbb{R}$,

- $\delta(k)=1, \quad \forall k \in[0, N]$.

The conditions $\left(\mathrm{H}_{0}\right)-\left(\mathrm{H}_{6}\right)$ are fulfilled.

Moreover we may consider $\mathrm{X}$ with the following norm

$$
|x|_{\mathrm{m}}=\left(\sum_{\mathrm{k}=1}^{\mathrm{N}}|x(\mathrm{k})|^{\mathrm{m}}\right)^{\frac{1}{\mathrm{~m}}}, \quad \forall x \in X \quad \text { and } \mathrm{m} \geq 2 .
$$

We have the following inequalities (see [3, 9])

$$
\mathrm{N}^{(2-\mathrm{m}) /(2 \mathrm{~m})}|x|_{2} \leq|x|_{\mathrm{m}} \leq \mathrm{N}^{1 / \mathrm{m}}|x|_{2}, \quad \forall x \in X \quad \text { and } \quad \mathrm{m} \geq 2
$$


We defined the convex modular $\rho: \mathrm{X} \longrightarrow \mathbb{R}$ by

$$
\rho(x)=\sum_{k=1}^{N+1}|\Delta x(k-1)|^{p(k-1)}
$$

and we introduce the Luxembourg norm

$$
\|x\|_{p(.)}=\inf \{\lambda>0: \rho(x / \lambda) \leq 1\} .
$$

Since $X$ has a finite dimension there exist constants $c_{5}>0, c_{6}>1$ such that

$$
c_{5}\|x\|_{p(.)} \leq\|x\| \leq c_{6}\|x\|_{p(.)} .
$$

The followings inequalities holds:

$$
\min \left\{\|x\|_{\mathfrak{p}(.)}^{\mathfrak{p}^{-}} ;\|x\|_{\mathfrak{p}(.)}^{\mathfrak{p}^{+}}\right\} \leq \rho(x) \leq \max \left\{\|x\|_{\mathfrak{p}(.)}^{\mathfrak{p}^{-}} ;\|x\|_{\mathfrak{p}^{(.)}}^{\mathbf{p}^{+}}\right\} .
$$

We need the following auxiliary results throughout our paper (see [5]).

\section{Lemma 2.2.}

(1) For every $x \in X$ and for every $m \geq 2$ we have

$$
\sum_{k=1}^{N+1}|\Delta x(k-1)|^{m} \leq 2^{m} \sum_{k=1}^{N}|x(k)|^{m}
$$

and

$$
\sum_{k=1}^{N+1}|\Delta x(k-1)|^{m} \geq(N+1)^{\frac{2-m}{2}}\|x\|^{m}
$$

(2) For every $x \in X$ and for every $m>1$ we have

$$
\sum_{k=1}^{N}|x(k)|^{m} \leq N(N+1)^{m-1} \sum_{k=1}^{N+1}|\Delta x(k-1)|^{m} .
$$

(3) For every $\mathrm{x} \in \mathrm{X}$ and for every $\mathrm{m} \geq 1$ we have

$$
\sum_{k=1}^{N+1}|\Delta x(k-1)|^{m} \leq(N+1)\|x\|^{m} .
$$

(4) For every $x \in X$ we have

$$
\sum_{k=1}^{N+1}|\Delta x(k-1)|^{p(k-1)} \leq(N+1)\|x\|^{p^{+}}+(N+1) .
$$


Definition 2.3. A $C^{1}$ functional J satisfies the Palais-Smale condition (in short (PS) condition) if any sequence $\left\{u_{n}\right\} \subset X$ such that $\left\{J\left(u_{n}\right)\right\}$ is bounded and

$$
\mathrm{J}^{\prime}\left(\mathrm{u}_{\mathrm{n}}\right) \longrightarrow 0, \quad \text { as } \quad \mathrm{n} \longrightarrow \infty
$$

has a convergent subsequence.

From (PS) condition, it follows that the set of critical points for a bounded functional is compact. This condition was a base for the modern development of critical point theory. It is needed for the mountain pass lemma (see [11]).

Lemma 2.4. Let $\mathrm{X}$ be a real reflexive Banach space. Assume that $\mathrm{J} \in \mathrm{C}^{1}(\mathrm{X}, \mathbb{R})$ and $\mathrm{J}$ satisfies the $(P S)$ condition. Suppose also that:

(1) $\mathrm{J}(0)=0$,

(2) there exist $\rho>0$ and $\alpha>0$ such that $\mathrm{J}(\mathrm{u}) \geq \alpha$ for all $\mathrm{u} \in \mathrm{X}$ with $\|\mathrm{u}\|=\rho$,

(3) there exists $\mathfrak{u}_{1} \in \mathbf{X}$ with $\left\|\mathfrak{u}_{1}\right\|>\rho$ such that $\mathrm{J}\left(\mathfrak{u}_{1}\right)<\alpha$.

Then, J has a critical value $\mathrm{c} \geq \alpha$. Moreover, $\mathrm{c}$ can be characterized as

$$
\inf _{\mathbf{g} \in \Gamma}\left(\max _{\mathfrak{u} \in \mathbf{g}([0,1])} J(\mathfrak{u})\right)
$$

where $\Gamma=\left\{\mathrm{g} \in \mathrm{C}([0,1], X): \mathrm{g}(0)=0, \mathrm{~g}(1)=\mathfrak{u}_{1}\right\}$.

Lemma 2.5 ([10, Lemma 2.2). Let $\mathrm{X}$ be a finite dimensional Banach space and let $\mathrm{J} \in \mathrm{C}^{1}(\mathrm{X}, \mathbb{R})$ be an anti-coercive functional (namely, $\lim _{\|\mathrm{u}\|+\infty} \mathrm{J}(\mathrm{u})=-\infty$ ). Then $\mathrm{J}$ satisfies the $(P S)$ condition.

\section{Existence of nontrivial solutions}

We define the energy functional $\mathrm{J}: \mathrm{X} \longrightarrow \mathbb{R}$ by

$$
J(u)=\sum_{k=1}^{N+1} A(k-1, \Delta u(k-1))-\sum_{k=1}^{N} \delta(k) F(k, u(k), w)
$$

and we put

$$
\mathrm{I}(\mathrm{u})=\sum_{k=1}^{\mathrm{N}+1} \mathrm{~A}(\mathrm{k}-1, \Delta \mathrm{u}(\mathrm{k}-1)) \text { and } \Lambda(\mathrm{u})=\sum_{\mathrm{k}=1}^{\mathrm{N}} \delta(\mathrm{k}) \mathrm{F}(\mathrm{k}, \mathrm{u}(\mathrm{k}), w) .
$$

Lemma 3.1. The functional $\mathrm{J}$ is well defined on $\mathrm{X}$ and is of class $\mathrm{C}^{1}(\mathrm{X}, \mathbb{R})$ with the derivative given by

$$
\left\langle J^{\prime}(u), v\right\rangle=\sum_{k=1}^{N+1} a(k-1, \Delta u(k-1)) \Delta v(k-1)-\sum_{k=1}^{N} \delta(k) f(k, u(k), w) v(k),
$$

for all $u, v \in X$. 


\section{Proof}

We have $|\mathrm{J}(\mathrm{u})| \leq|\mathrm{I}(\mathrm{u})|+|\Lambda(\mathrm{u})|$.

Since $A(k,$.$) is continuous for all k \in[0, N]$, then

$$
|\mathrm{I}(\mathrm{u})|=\left|\sum_{k=1}^{N+1} A(k-1, \Delta \mathrm{u}(\mathrm{k}-1))\right|<+\infty .
$$

Also from (2.7) and Holder's inequality we have

$$
\begin{aligned}
|\Lambda(u)| & =\left|\sum_{k=1}^{N} \delta(k) F(k, u(k), w)\right| \\
& \leq \sum_{k=1}^{N}|\delta(k)||F(k, u(k), w)| \\
& \leq c_{2} \bar{\delta} \sum_{k=1}^{N}\left(1+|u(k)|^{r(k)}\right) \\
& \leq N c_{2} \bar{\delta}+c_{2} \bar{\delta} \sum_{k=1}^{N}|u(k)|^{r(k)} \\
& \leq 2 N c_{2} \bar{\delta}+c_{2} \bar{\delta} \sum_{k=1}^{N}|u(k)|^{r^{+}} \\
& \leq 2 N c_{2} \bar{\delta}+c_{2} \bar{\delta}\left(\left.\left.\sum_{k=1}^{N}|| u(k)\right|^{r^{+}}\right|^{\frac{2}{r+}}\right)^{\frac{r^{+}}{2}}\left(\sum_{k=1}^{N}|1|^{\frac{1}{1-\frac{r^{+}}{2}}}\right)^{1-\frac{r^{+}}{2}} \\
& \leq 2 N c_{2} \bar{\delta}+c_{2} \bar{\delta} N^{\frac{2-r^{+}}{2}}\left(\sum_{k=1}^{N}|u(k)|^{2}\right)^{\frac{r^{+}}{2}} \\
& <+\infty .
\end{aligned}
$$

Then, the energy functional $\mathrm{J}$ is well defined on $\mathrm{X}$.

As in 6], Lemma 3.4, we can prove that the functional I derivative is given by

$$
\left\langle\mathrm{I}^{\prime}(\mathrm{u}), v\right\rangle=\sum_{k=1}^{\mathrm{N}+1} \mathrm{a}(\mathrm{k}-1, \Delta \mathrm{u}(\mathrm{k}-1)) \Delta v(\mathrm{k}-1) .
$$


On the other hand, for all $u, v \in X$, we have

$$
\begin{aligned}
\left\langle\Lambda^{\prime}(u), v\right\rangle & =\lim _{h \rightarrow 0^{+}} \frac{\Lambda(u+h v)-\Lambda(u)}{h} \\
& =\lim _{h \rightarrow 0^{+}} \sum_{k=1}^{N} \delta(k) \frac{F(k, u(k)+h v(k), w)-F(k, u(k), w)}{h} \\
& =\sum_{k=1}^{N} \delta(k) \lim _{h \rightarrow 0^{+}} \frac{F(k, u(k)+h v(k), w)-F(k, u(k), w)}{h} \\
& =\sum_{k=1}^{N} \delta(k) f(k, u(k), w) .
\end{aligned}
$$

The functional $\mathrm{J}$ is clearly of class $\mathrm{C}^{1}$

Lemma 3.2. The functional $\mathrm{J}$ is anti-coercive.

\section{Proof}

Let $\|\mathrm{u}\|_{p_{(.)}}>1$. According to (2.3) and the inequality (2.14) there exist a constant $\mathrm{c}_{7}>0$ such that

$$
\begin{aligned}
I(u) & \leq c_{7}(N+1)+c_{7} \sum_{k=1}^{N+1}|\Delta u(k-1)|^{p(k-1)} \\
& \leq c_{7}(N+1)+c_{7}\|u\|_{p(.)}^{p^{+}}
\end{aligned}
$$

According to (2.9), (2.15) and (2.16) we have

$$
\begin{aligned}
-\Lambda(u) & =-\sum_{k=1}^{N} \delta(k) F(k, u(k), w) \\
& \leq-\sum_{k=1}^{N} \delta(k)\left(c_{3}|u(k)|^{\mu}-c_{4}\right) \\
& \leq-\underline{\delta} c_{3} \sum_{k=1}^{N}|u(k)|^{\mu}+c_{4} \underline{\delta} N \\
& \leq-\underline{\delta} c_{3} 2^{-\mu} \sum_{k=1}^{N+1}|\Delta u(k-1)|^{\mu}+c_{4} \underline{\delta} N \\
& \leq-\underline{\delta} c_{3} 2^{-\mu}(N+1)^{\frac{2-\mu}{2}}\|u\|^{\mu}+c_{4} \underline{\delta} N .
\end{aligned}
$$

Consequently, combining (3.4) and (3.5) we get

$$
\mathrm{J}(\mathrm{u}) \leq-\underline{\delta} \mathrm{c}_{3} 2^{-\mu}(\mathrm{N}+1)^{\frac{2-\mu}{2}}\|u\|^{\mu}+\mathrm{c}_{4} \underline{\delta} \mathrm{N}+\mathrm{c}_{7}\|\mathrm{u}\|_{\mathrm{p}(.)}^{p^{+}}+\mathrm{c}_{7}(\mathrm{~N}+1) .
$$


Since the norms $\|\cdot\|$ and $\|\cdot\|_{p(.)}$ are equivalents and since $\mu>p^{+}$then the functional $J$ is anticoercive

We can deduce from Lemma 2.5 that the function J satisfies the (PS) condition.

Theorem 3.3. For any $w \in W$, the problem (1.2) has at least one non trivial solution.

\section{Proof}

According to $\left(\mathrm{H}_{4}\right)$, for any $\varepsilon>0$ there exists $\beta>0$ such that for all $\xi \in[-\beta, \beta], \quad|f(k, \xi, w)| \leq$ $\varepsilon|\xi|^{p^{+}-1}$ for all $k \in[1, N], \quad w \in W$.

As in [10, it is obvious to see that for any $\varepsilon \in\left(0, \frac{\left(N+1 \frac{2-p^{+}}{2}\right.}{\delta \mathrm{N}(\mathrm{N}+1)^{\mathrm{p}^{+}}}\right)$there exists $\beta>0$ such that for all $x \in[-\beta, \beta]$ we have

$$
|\mathrm{F}(\mathrm{k}, \xi, w)| \leq \varepsilon \frac{|\xi|^{\mathrm{p}^{+}}}{\mathrm{p}^{+}}, \quad \forall \mathrm{k} \in[1, \mathrm{~N}], w \in \mathrm{W} .
$$

Let $u \in X \cap\{|u(k)| \leq \beta, \forall k \in[1, N]\} \cap\{\|u\| \leq 1\}$. We have $\|u\| \leq 2 \beta(N+1)^{\frac{1}{2}}$ and according to (2.16) we have

$$
\sum_{k=1}^{N+1}|\Delta u(k-1)|^{p(k-1)} \geq \sum_{k=1}^{N+1}|\Delta u(k-1)|^{p^{+}} \geq(N+1)^{\frac{2-p^{+}}{2}}\|u\|^{p^{+}} .
$$

If we put $\eta=\min \left\{1 ; 2 \beta(N+1)^{\frac{1}{2}}\right\}$, then for any $u \in X$ with $\|u\| \leq \eta$, by (3.7), (3.8), $\left(\mathrm{H}_{2}\right)$, (2.17) and (2.18) it follows that

$$
\begin{aligned}
J(u) & \geq \frac{1}{p^{+}} \sum_{k=1}^{N+1}|\Delta u(k-1)|^{p(k-1)}-\bar{\delta} \varepsilon \frac{1}{p^{+}} \sum_{k=1}^{N}|u(k)|^{p^{+}} \\
& \geq \frac{1}{p^{+}}(N+1)^{\frac{2-p^{+}}{2}}\|u\|^{p^{+}}-\bar{\delta} \varepsilon \frac{1}{p^{+}} N(N+1)^{p^{+}}\|u\|^{p^{+}} \\
& \geq\|u\|^{p^{+}} \frac{1}{p^{+}}\left((N+1)^{\frac{2-p^{+}}{2}}-\bar{\delta} \varepsilon N(N+1)^{p^{+}}\right) .
\end{aligned}
$$

Remark that $\varepsilon$ is such that $(\mathrm{N}+1)^{\frac{2-\mathrm{p}^{+}}{2}}-\bar{\delta} \varepsilon \mathrm{N}(\mathrm{N}+1)^{\mathrm{p}^{+}}>0$. So there exists positive numbers $0<\rho=\|u\| \leq \eta$ and

$$
\alpha=\frac{\rho^{p^{+}}}{p^{+}}\left((N+1)^{\frac{2-p^{+}}{2}}-\bar{\delta} \varepsilon N(N+1)^{p^{+}}\right)>0
$$

such that $\mathrm{J}(\mathrm{u}) \geq \alpha$ for all $u \in X$ with $\|u\|=\rho$. Also we have $J(0)=0$ and since $J$ is anti-coercive, there exists $u_{1} \in X$ with $\left\|u_{1}\right\|>\rho$ such that $J\left(u_{1}\right)<\alpha$.

By the mountain pass lemma (see Lemma 2.4), the functional $J$ has a critical value $c^{*}>0$, i.e, there exists $u^{*} \in X$ such that $J\left(u^{*}\right)=c^{*}$ and $\left\langle J^{\prime}\left(u^{*}\right), v\right\rangle=0, \forall v \in X$.

Since $J(0)=0$ it is obvious to see that $u^{*} \neq 0$. The critical value $c^{*}$ can be characterized as

$$
\mathrm{c}^{*}=\inf _{v \in \Gamma}\left(\max _{\mathrm{t} \in[0,1]} \mathrm{J}(v(\mathrm{t}))\right),
$$


where $\Gamma=\left\{v \in \mathrm{C}([0,1], \mathrm{X}): v(0)=0, v(1)=u_{1}\right\}$. Then, the proof of the existence of solution to problem (1.2) for any parameter $w \in W$ is complete

Now, in what follow, we examine conditions under which our problem (1.2) has a unique non trivial solution. Assume that for any $k \in[0, N], \xi_{1}, \xi_{2} \in \mathbb{R}$ and $w \in W$ the followings additional conditions are satisfies:

$\left(\mathrm{H}_{7}\right)$. there exist a constant $\mathrm{c}_{8}>0$ such that

$$
\left(a\left(k, \xi_{1}\right)-a\left(k, \xi_{2}\right)\right) \geq c_{8}\left|\xi_{1}-\xi_{2}\right|^{p^{+}},
$$

$\left(\mathrm{H}_{8}\right)$. there exist a constant $0<\mathrm{d}<\frac{\mathrm{c}_{8}}{\bar{\delta} \mathrm{N}(\mathrm{N}+1)^{\frac{3 p^{+}-2}{2}}}$ such that

$$
\left|f\left(k, \xi_{1}, w\right)-f\left(k, \xi_{2}, w\right)\right| \leq d\left|\xi_{1}-\xi_{2}\right|^{p^{+}-1} .
$$

Theorem 3.4. If $\mathrm{u} \in \mathrm{X}$ is a non trivial solution of problem (1.2), if $\left(\mathrm{H}_{7}\right)$ and $\left(\mathrm{H}_{8}\right)$ are holds, then for every fixed parameter $w \in \mathrm{W}$, the solution $\mathrm{u}$ is unique.

\section{Proof}

Let $u, v \in X$ two nonzero solutions of problem (1.2). Then

$$
\sum_{k=1}^{N+1} a(k-1, \Delta u(k-1)) \Delta(u-v)(k-1)=\sum_{k=1}^{N} \delta(k) f(k, u(k), w)(u-v)(k)
$$

and

$$
\sum_{k=1}^{N+1} a(k-1, \Delta v(k-1)) \Delta(v-u)(k-1)=\sum_{k=1}^{N} \delta(k) f(k, v(k), w)(v-u)(k) .
$$

Upon addition, we get

$$
\begin{aligned}
\sum_{k=1}^{N+1}[a(k-1, \Delta u(k-1)) & -a(k-1, \Delta v(k-1))] \Delta(u-v)(k-1) \\
= & \sum_{k=1}^{N} \delta(k)[f(k, u(k), w)-f(k, v(k), w)](u-v)(k) .
\end{aligned}
$$

Therefore, according to $\left(\mathrm{H}_{7}\right)$ and $\left(\mathrm{H}_{8}\right)$ we have

$$
c_{8} \sum_{k=1}^{N+1}|\Delta(u-v)(k-1)|^{p^{+}} \leq d \bar{\delta} \sum_{k=1}^{N}|(u-v)(k)|^{p^{+}} .
$$

Since $\mathrm{p}^{+} \geq 2$, using the inequalities (2.16)-(2.18) we can write

$$
\begin{aligned}
c_{8}(N+1)^{\frac{2-p^{+}}{2}}\|u-v\|^{p^{+}} & \leq \mathrm{d} \bar{\delta} N(N+1)^{p^{+}-1} \sum_{k=1}^{N+1}|\Delta(u-v)(k-1)|^{p^{+}} \\
& \leq d \bar{\delta} N(N+1)^{p^{+}}\|u-v\|^{p^{+}}
\end{aligned}
$$


namely

$$
\left[\frac{c_{8}}{\bar{\delta} N(N+1)^{\frac{3 p^{+}-2}{2}}}-d\right]\|u-v\|^{p^{+}} \leq 0 \text {. }
$$

Recall that the constant $d$ is such that $\frac{c_{8}}{\bar{\delta} N(N+1)^{\frac{3 p^{+}-2}{2}}}-d>0$. Consequently,

$$
\|\mathfrak{u}-v\|^{p^{+}}=0, \quad \text { thus } \quad \mathfrak{u}=v
$$

\section{References}

[1] R. P. Agarwal, K. Perera and D. O'Regan; Multiple positive solutions of singular and nonsingular discrete problems via variational methods, Nonlinear Anal. 58 (2004), 69-73.

[2] A. Cabada, A. Iannizzoto and S. Tersian; Multiple solutions for discrete boundary value problems. J Math Anal Appl. 356 (2009), 418-428.

[3] X. Cai and J. Yu; Existence theorems for second-order discrete boundary value problems, J. Math. Anal. Appl. 320 (2006), 649-661.

[4] P. Candito and G. D'Agui; Three solutions for a discrete nonlinear Neumann problem involving p-Laplacian, Adv Differ Equ 11 (2010). Article ID 862016

[5] M. Galewski and R. Wieteska; Existence and multiplicity of positive solutions for discrete anisotropic equations, Turk. J. Math. 38 (2014), 297-310.

[6] A. Guiro, I. Nyanquini and S. Ouaro; On the solvability of discrete nonlinear Neumann problems involving the $\mathrm{p}(\mathrm{x})$-Laplacian, Adv. Differ. equ. 32 (2011).

[7] L. Jiang and Z. Zhou; Three solutions to Dirichlet boundary value problems for $\mathrm{p}$-Laplacian Difference equations, Adv Differ Equ 10 (2008). Article ID 345916.

[8] B. Koné and S. Ouaro; Weak solutions for anisotropic discrete boundary value problems. J Differ Equ Appl. 16(2) (2010), 1-11.

[9] M. Mihailescu, V. Radulescu and S. Tersian; Eigenvalue problems for anisotropic discrete boundary value problems, J. Differ. Equ. Appl. 15 (2009), 557-567.

[10] J. Smejda and R. Wieteska; On the dependence on parameters for second order discrete boundary value problems with the $\mathrm{p}(\mathrm{k})$-laplacian, Opuscula Math. 34 (2014), 851-870.

[11] M. Willem; Minimax Theorem, Birkhuser, 1996.

[12] J. Yu and Z. Guo; On boundary value problems for a discrete generalized Emden-Fowler equation. J Math Anal Appl. 231 (2006), 18-31.

[13] G. Zhang and S. Liu; On a class of semipositone discrete boundary value problem. J Math Anal Appl. 325 (2007), 175-182. 